\title{
Rapid detection of 2,2',4,4'-tetrabromodiphenyl ether (BDE-47) using a portable Au-colloid SERS sensor
}

\author{
Zhenli Sun, Jingjing Du, Li Yan and Chuanyong Jing*
}

In situ rapid detection and identification of polybrominated diphenyl ethers, a group of well-known persistent organic pollutants, present a great challenge. To develop a portable and sensitive surface-enhanced Raman scattering (SERS) sensor for rapid 2,2',4,4'-tetrabromodiphenyl ether (BDE-47) detection, we adopted the most commonly used Au nanoparticles, which are effective in the analysis of hydrophobic BDE-47 with a simple optimization in citrate content and sampling technique. Qualitative and quantitative determination of BDE-47 was achieved using a portable Raman spectrometer. The SERS response exhibited a linear dependence on the BDE-47 concentration up to $1000 \mathrm{nM}$ with a detection limit of $75 \mathrm{nM}$. The density function theory-calculated Raman spectra agreed well with the experimental observations, and the results justified the existence of electromagnetic enhancement and charge transfer mechanism. This in situ SERS platform allows easy and reliable detection of hydrophobic molecules such as BDE-47 in complex matrices. Copyright @ 2014 John Wiley \& Sons, Ltd.

Additional supporting information may be found in the online version of this article at the publisher's web site.

Keywords: SERS; DFT; PBDEs; hydrophobic molecules; portable sensor

\section{Introduction}

As the most widely spread and toxic polybrominated diphenyl ethers congener in the environment, 2,2',4,4'-tetrabromodiphenyl ether (BDE-47) presents an increasing public concern. ${ }^{[1]}$ Identification and quantification of BDE-47 usually involve rigorous sample pre-treatment before analysis with gas chromatography/mass spectrometry. These instruments are not designed for field application. Therefore, the motivation of our research is to develop a portable and sensitive sensor for rapid BDE-47 detection.

Surface-enhanced Raman scattering (SERS) provides a rapid and efficient platform for chemical analysis due to the explosive development in nanofabrication. ${ }^{[2,3]}$ Compared with the traditional chromatographic methods, SERS can selectively identify single component in complex matrices due to the specific close contact between the analyte and noble metal nanoparticles (NPs). Unfortunately, the hydrophobic nature of polycyclic aromatic hydrocarbons, polychlorinated biphenyls (PCBs), and polybrominated diphenyl ethers prevents their approach to the metallic nanostructure. Consequently, numerous efforts have been focused on functionalizing metal surfaces to capture hydrophobic molecules. ${ }^{[4-9]}$ For instance, the alkanethiol-modified Ag NPs were used for SERS sensing of BDE-15. ${ }^{[7]}$ Although this method could achieve SERS detection of BDE-15, the stringent and complicated protocol is a prerequisite for the successful synthesis of substrate.

A challenge for the SERS analysis of hydrophobic molecules is to obtain pronounced SERS enhancements using readily available NPs without stringent structural requirements nor special functionality. The citrate-reduced Au NP colloid is such a versatile SERS substrate, which is reproducible, sensitive, and selective. ${ }^{[10]}$ Our hypothesis is that this commonly used Au NPs could be effective in the analysis of hydrophobic BDE-47 with a simple optimization in citrate content and sampling technique.
The objective of this study was to develop a portable SERS sensor for rapid BDE-47 detection. The Au NPs were obtained using a one-pot synthesis based on the traditional citrate reduction approach and resulted in an enhancement factor (EF) of $10^{6}$. The density function theory (DFT) calculations were employed to justify the SERS peak assignments and the enhancement mechanism. Our results open a new venue to SERS application in the analysis of hydrophobic environmental pollutants.

\section{Experimental}

\section{Chemicals and materials}

All reagents were of analytical reagent grade and used without further purification. $\mathrm{HAuCl}_{4} 4 \mathrm{H}_{2} \mathrm{O}$ and trisodium citrate dehydrate were purchased from Sinopharm Chemical Reagent Co., Ltd (China). BDE-47, pyrene, and 2,2',4,4'-tetrachlorobiphenyl (PCB47) were from J\&K Scientific Ltd. (China). Suwannee River humic acid $(\mathrm{HA})$, (catalog no. $2 \mathrm{~S} 101 \mathrm{H}$ ) was from the International Humic Substances Society (IHSS). Absolute ethanol (>99.7\%) was from Beijing Chemical Reagents Company (China). Milli-Q water $(>18.2 \mathrm{M} \Omega)$ was used in all experiments. The stock solution of $\mathrm{HAuCl}_{4}$ (2 wt.\% in water) was prepared by dissolving $\mathrm{HAuCl}_{4} 4 \mathrm{H}_{2} \mathrm{O}$ in Milli-Q water and stored in a brown bottle at $4^{\circ} \mathrm{C}$.

\footnotetext{
* Correspondence to: Chuanyong Jing, State Key Laboratory of Environmental Chemistry and Ecotoxicology, Research Center for Eco-Environmental Sciences, Chinese Academy of Sciences, Beijing 100085, China. E-mail: cyjing@rcees.ac.cn
}

State Key Laboratory of Environmental Chemistry and Ecotoxicology, Research Center for Eco-Environmental Sciences, Chinese Academy of Sciences, Haidian District, Beijing, China 


\section{One-step synthesis of gold nanoparticles}

All glassware and stirring paddle Polytetrafluoroethene were rigorously cleaned by aqua regia $\left(\mathrm{HCl} / \mathrm{HNO}_{3}=3: 1, \mathrm{v} / \mathrm{v}\right)$, followed by a thorough rinsing with Milli-Q water. The citrate-stabilized Au NPs were synthesized by modifying the procedure described by literature. ${ }^{[11]}$ Briefly, a total of $1 \mathrm{~mL}$ of $\mathrm{HAuCl}_{4}$ (2 wt. \%) was added to $23-\mathrm{mL} \mathrm{H}_{2} \mathrm{O}$ in a three-neck flask and heated to $100^{\circ} \mathrm{C}$ in an oil bath with stirring. Then, $1-\mathrm{mL}$ sodium citrate $(40-100 \mathrm{mg})$ was quickly added to the aforementioned solution. The mixture was reacted for $15 \mathrm{~min}$ at $100^{\circ} \mathrm{C}$. The resulted colloid was cooled at room temperature under vigorous stirring.

\section{Sample preparation for SERS analysis}

The samples of BDE-47, PCB-47, pyrene, and HA were prepared by diluting the stock solution in DI water, river water, groundwater, and aged-tap water to reach a final concentration of $1 \mathrm{mg} / \mathrm{L}$ for $\mathrm{HA}$ and $1 \times 10^{-6} \mathrm{M}$ for others. The samples $(0.5 \mathrm{~mL})$ were mixed with the $\mathrm{Au} N \mathrm{NP}$ colloid $(0.5 \mathrm{~mL})$ and then centrifuged at $8000 \mathrm{rpm}$ for $20 \mathrm{~min}$. About $10-\mu \mathrm{L}$ solution was placed on a silicon wafer and subjected to SERS measurements.

\section{Apparatus}

The UV-vis spectra were recorded on a SHIMADZU UV-2550 spectrophotometer. The transmission electron microscopy (TEM) analysis was performed on JEM-1400 from JEOL Ltd. Raman spectra were obtained using a portable Raman spectrometer (Enwave Optronics, Inc. USA) with a $4 \mathrm{~cm}^{-1}$ resolution at the excitation energy of $785 \mathrm{~nm}$.

\section{DFT calculations}

All quantum chemical calculations were performed with the program package Gaussian 09 with B3LYP hybrid DFT. A series of clusters containing increasing numbers of Au atoms from 1 to 10 were structural optimized and then used to calculate the Raman shift. The 6-31G basis set was used for $C, H$, and O. The $6-311+G(d, p)$ was used for $B r$ and LANL2DZ for Au. To correct the well-known overestimations of the calculated wavenumbers, all the calculated frequencies of BDE-47-Aun $(n=0-10)$ were scaled by a factor of 0.9614 .

\section{Results and discussion}

\section{Optimization of citrate content in Au NPs preparation}

To determine the optimal citrate content in the synthesis of $\mathrm{Au}$ NPs for BDE-47 analysis, the molar ratio of citrate to $\mathrm{HAuCl}_{4}$ in the range from 3 to 8 was investigated, and the results are shown in Fig. 1. The SERS intensity of BDE-47 at $665 \mathrm{~cm}^{-1}$ reached the maximum as the ratio increased from 3 to 6 [Fig. 1(I)]. This increase in peak intensity corresponded well to the aggregation of Au NPs as evidenced in the UV-vis spectra and TEM images [Fig. 1(II, III)]. In agreement with our observations, the moderate aggregates of NPs have been attributed to the enhanced SERS response due to the increase in hot spots under close proximity of NPs. ${ }^{[12]}$ However, the SERS intensity decreased when the ratio was greater than 6 . Correspondingly, the strong and sharp peak in UV-vis spectra became weak and broad [Fig. 1(II)]. In addition, the color of the Au colloid turned from wine red to pale pink [inset of Fig. 1(III)], indicating the intensive aggregation of Au NPs. Therefore, the molar ratio of citrate to $\mathrm{HAuCl}_{4}$ was set at 6 to prepare the Au NPs. The resulting NP exhibited an absorption peak at $523 \mathrm{~nm}$ in the UV-vis spectrum [Fig. 1(II)], the ratio of peak intensity at this surface plasma resonance $(523 \mathrm{~nm})$ to that at $450 \mathrm{~nm}$ was 1.69 , suggesting the Au NP size of $18 \mathrm{~nm} .{ }^{[13]}$ This particle size was confirmed by the TEM analysis.

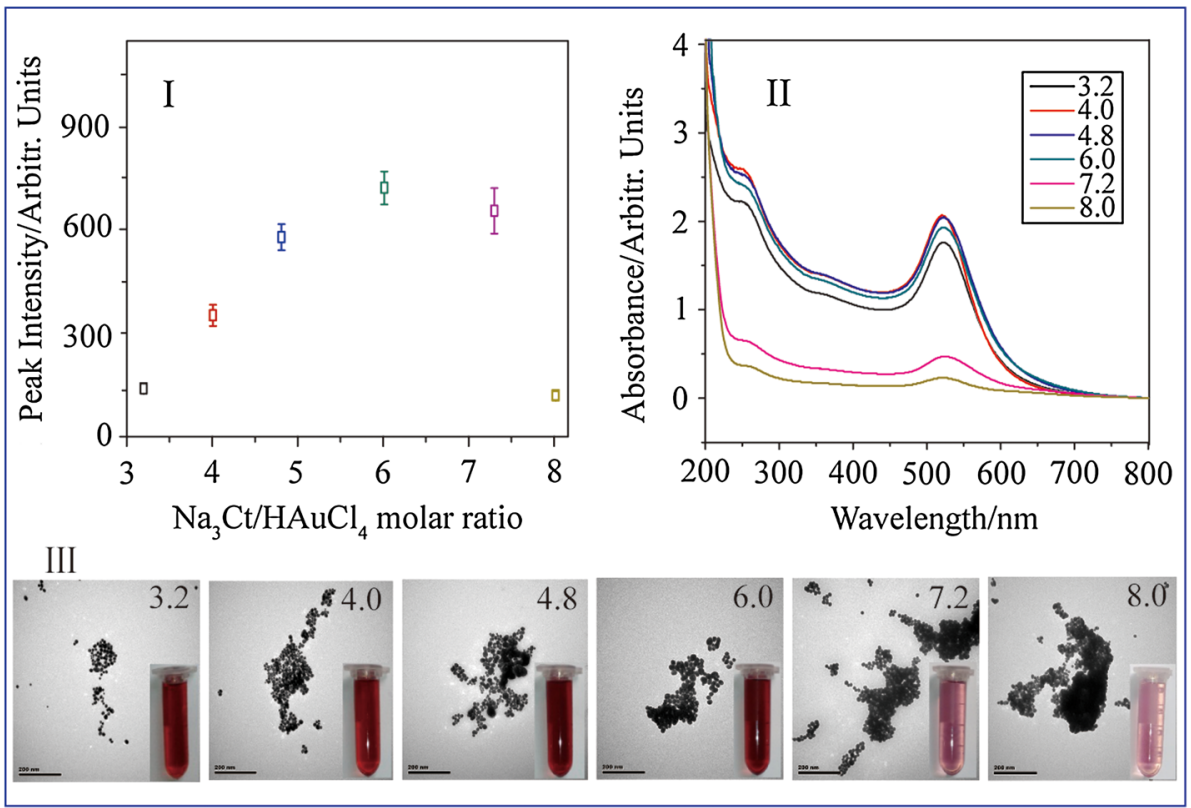

Figure 1. (I) Peak intensity of $2.5 \times 10^{-7} \mathrm{M} \mathrm{BDE}-47$ at $665 \mathrm{~cm}^{-1}$ as a function of citrate/ $\mathrm{HAuCl}_{4}$ molar ratio, the data represent the average \pm standard deviation at 25 randomly chosen points, signal collecting time $=5 \mathrm{~s}$. (II) UV-vis spectrum of the Au NPs with the molar ratio increased from 3 to 8 . (III) TEM images with the molar ratio ranged from 3 to 8 , the scale bars represent $200 \mathrm{~nm}$. The inset photograph shows the Au NP colloid at each ratio. 


\section{Effect of sampling preparation sequence}

To find the optimal sampling preparation technique, two sampling preparation sequences were adopted as illustrated in the inset of Fig. 2: in one, the sample was mixed with the Au colloid before centrifugation, i.e. MC and in the other, centrifugation before mixing, i.e., CM. The SERS intensity at $665 \mathrm{~cm}^{-1}$ was used to compare the two sequences (Fig. 2). The results clearly show that $\mathrm{MC} 10$, i.e., $\mathrm{MC}$ with 10 times concentrating, exhibited the maximum intensity (2555). In contrast, CM process did not result in any SERS signal up to five times concentrated, and the intensity was only about 115 up to 10 times concentrated (Fig. 2). The centrifugal process promotes the BDE-47 molecules approaching to the Au NPs and subsequently facilitates their close contact with the 'hot spots' to provide huge SERS enhancements. Therefore, MC10 was used as the high activity sample preparation method.

\section{Quantitative analysis of BDE-47}

To validate the effectiveness of our method, BDE-47 at different concentrations were detected by the way mentioned earlier. The SERS intensity of the most intensive band at $665 \mathrm{~cm}^{-1}$ was recorded from 25 randomly selected spots and used to quantify the SERS response as a function of BDE-47 concentration (Fig. 3). The intensity increased linearly with concentration increasing from 75 to $1000 \mathrm{nM}\left[R^{2}=0.97\right.$, Fig. 3(II)]. The detection limit was determined to be about $75 \mathrm{nM}$ [Fig. 3(I)]. The EF was $10^{6}$ calculated using the following equation: $\mathrm{EF}=\left(I_{\mathrm{SERS}} / I_{\mathrm{RS}}\right),\left(C_{\mathrm{RS}} / C_{\mathrm{SERS}}\right)$, where $I_{\text {SERS }}$ is the SERS intensity at $665 \mathrm{~cm}^{-1} ; I_{\mathrm{RS}}$ is the normal Raman intensity at $665 \mathrm{~cm}^{-1}$; and $C_{\mathrm{RS}} / C_{\mathrm{SERS}}$ is the BDE-47 concentration ratio in normal Raman over SERS sample. ${ }^{[14]}$ The positions and assignments of Raman shifts for BDE-47 are listed [Fig. 3(I) and Table 1). Five-weak bands at 801, 1024, 1318, 1380 , and $1602 \mathrm{~cm}^{-1}$ were attributable to citrate and its decomposition products. ${ }^{[15]}$

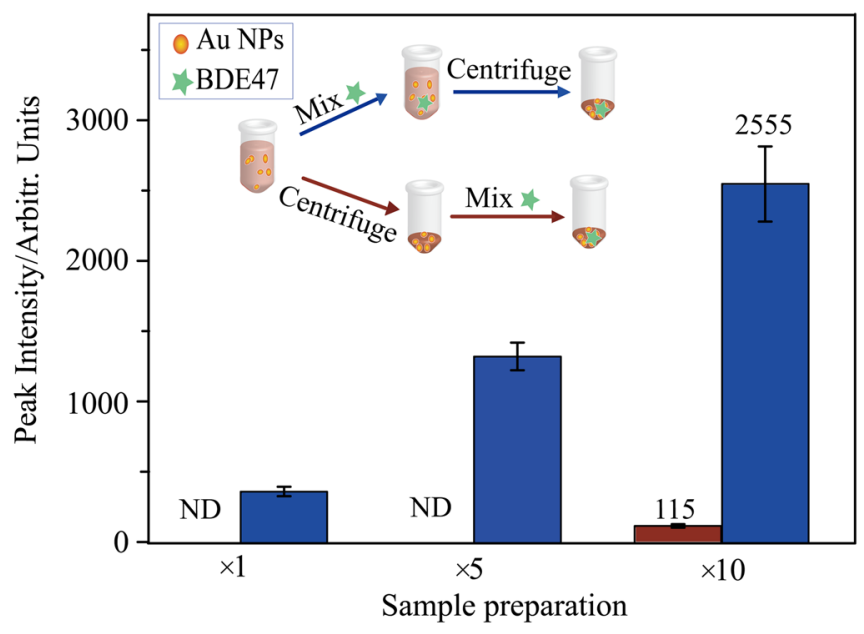

Figure 2. SERS intensity (peak height at $665 \mathrm{~cm}^{-1}$ ) for $1 \times 10^{-6} \mathrm{M} \mathrm{BDE}-47$ prepared by different methods. MC means mix BDE-47 with Au colloid before centrifugation, and $\mathrm{CM}$ means centrifugation $\mathrm{Au}$ colloid before mixing. The samples were concentrated by 1,5 , and 10 times. The data points represented the average \pm standard deviation for 25 randomly chosen points. Signal collecting time $=5 \mathrm{~s}$. The inset shows the sampling process for different detection methods.

\section{SERS peak assignment and enhancement mechanism}

To explore the SRES enhancement mechanism of BDE-47, quantum chemical calculation with DFT was employed to predict the molecular structure and Raman spectra for BDE$47-\mathrm{Au}_{\mathrm{n}} \quad(n=0-10)$. The observed and calculated peak positions and corresponding assignments are summarized in Table 1.

Generally, both electromagnetic and charge-transfer (CT) effects contribute to the overall SERS enhancement. ${ }^{[16]}$ The primary enhancement $\left(10^{5}-10^{6}\right)$ is usually because of the physical electromagnetic mechanism, with a small contribution from the chemical CT enhancement $\left(10^{3}\right) .{ }^{[17]}$ The former does not involve the formation of chemical bonds between the analyte and substrate, and thus the peak position does not significantly shift. As shown in Fig. 4(I) and Table 1, the DFT-calculated peak position of BDE-47- $\mathrm{Au}_{n}$ agreed well with the experimental observations and the calculated wavenumber of skeletal stretch $\left(662-664 \mathrm{~cm}^{-1}\right)$ was in line with the observed band at $672 \mathrm{~cm}^{-1}$ (Fig. S1).

When a BDE-47 molecule adsorbs the Au clusters, an additional Au-BDE-47 CT effect might occur because of the interaction between the molecule and the Au cluster. Indeed, the DFT results show that the Mulliken charge transferred from $B D E-47$ to $A u$ ranged from 0.30 to 0.60 electrons in different $\mathrm{Au}_{\mathrm{n}}(n=0-10)$ clusters (Table S1). The same CT direction was also reported when PCB-52 adsorbed on Ag clusters. ${ }^{[18]}$ The $\mathrm{CT}$ accompanied different $\mathrm{Au}-\mathrm{Br}$ bond lengths ranging 2.6 to $3.1 \AA$ (Table S1). The CT can further be justified with the energy state analysis of the highest occupied molecular orbital and the lowest unoccupied molecular orbital (LUMO). The results in Fig. 4(II) show that the energy of the electronic transition states varied in the range $0.07-0.20 \mathrm{eV}$, which allowed the $\mathrm{CT}$ under the irradiation of $785 \mathrm{~nm}$ laser $(1.66 \mathrm{eV})$.

According to the CT mechanism of SERS, the peaks are selectively enhanced when excited by different laser lines. ${ }^{[19]}$ Moreover, the CT mechanism depends sensitively on the particular chemical bonds between the analyte and the substrate, resulting in selective enhancement of peaks. In this study, SERS spectra of $1 \times 10^{-6} \mathrm{M}$ BDE-47 were recorded with two different excitation wavelengths, 633 and $785 \mathrm{~nm}$. As shown in Fig. S2, the SERS intensity of BDE-47 was related to the excitation wavelength, i.e. the peak intensity was selectively enhanced. Our experimental results agree well with DFT calculations and suggest the existence of the $\mathrm{CT}$ mechanism in BDE-47 SERS enhancement.

\section{Identification of BDE-47 in complex matrices}

To investigate the SERS selectivity for BDE-47 in complex environmental matrices, $\mathrm{HA}, \mathrm{BDE}-47$, and structural similar compounds such as pyrene and PCB-47 were analyzed in DI water, groundwater, tap water, and river water matrices (Fig. 5). As shown in Fig. 5, the key SERS peaks of each individual hydrophobic pollutant can be readily identified. For example, PCB-47 exhibited a weak characteristic peak at $688 \mathrm{~cm}^{-1}$ in DI water. ${ }^{[8]}$ This obvious difference in peak enhancement between BDE-47 and PCB-47 could be mainly caused by their different functional groups on the benzene ring. For BDE-47, our DFT calculation indicated that the stable complexes were formed when $\mathrm{Br}$ atoms of $\mathrm{BDE}-47$ 

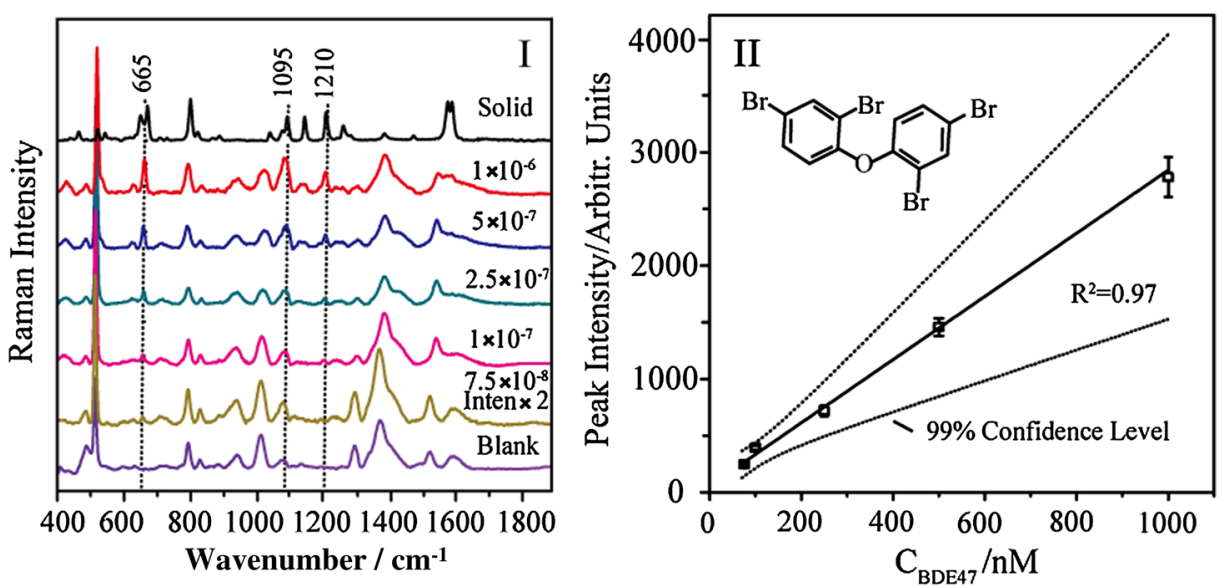

Figure 3. (I) Raman spectra of BDE47 with concentrations from $7.5 \times 10^{-8} \mathrm{M}$ to $1 \times 10^{-6} \mathrm{M}$. Signal collecting time was $10 \mathrm{~s}$ for the $7.5 \times 10^{-8} \mathrm{M} \mathrm{BDE}-47$ and $5 \mathrm{~s}$ for other concentrations. (II) Calibration curve for BDE-47 from 75 to $1000 \mathrm{nM}$. The calibration uses the most intensive SERS band at $665 \mathrm{~cm}^{-1}$. The data points represented the average \pm standard deviation for 25 randomly chosen points.

\begin{tabular}{|c|c|c|}
\hline Experimental & Calculated & Assignment \\
\hline 463 & 458 & Skeletal rocking \\
\hline 543 & 539 & Skeletal stretching \\
\hline 652 & 635 & Skeletal stretching \\
\hline 672 & 664 & Skeletal stretching \\
\hline 711 & 721 & $\mathrm{C}-\mathrm{C}$ stretching; $\mathrm{CH}$ in-plane bending \\
\hline 803 & 790 & $\mathrm{CH}$ wagging \\
\hline 891 & 871 & $\mathrm{CH}$ wagging \\
\hline 1044 & 1041 & $\mathrm{C}-\mathrm{C}$ stretching; $\mathrm{CH}$ in-plane bending \\
\hline 1095 & 1082 & $\mathrm{C}-\mathrm{C}$ breathing; $\mathrm{CH}$ in-plane bending \\
\hline 1146 & 1147 & $\mathrm{CH}$ in-plane bending \\
\hline 1210 & 1197 & $\mathrm{CH}$ in-plane bending \\
\hline 1261 & 1247 & $\mathrm{C}-\mathrm{C}$ breathing; $\mathrm{CH}$ in-plane bending \\
\hline 1282 & 1263 & $\mathrm{C}-\mathrm{Br}$ stretching; $\mathrm{CH}$ in-plane bending \\
\hline 1385 & 1371 & $\mathrm{C}-\mathrm{C}$ stretching; $\mathrm{CH}$ in-plane bending \\
\hline 1471 & 1468 & $\mathrm{C}-\mathrm{C}$ stretching; $\mathrm{CH}$ in-plane bending \\
\hline 1573 & 1583 & $\mathrm{C}-\mathrm{C}$ stretching \\
\hline
\end{tabular}

were closely coordinated with Au clusters as evidenced by their 2.6-3.1- $\AA$ atomic distances (Table S2). Similarly, close association of $\mathrm{Ag}-\mathrm{Cl}$ atoms was observed in the $\mathrm{PCB}-52 / \mathrm{Ag}_{\mathrm{n}}$ $(n=0-10)$ system, and the calculated $\mathrm{Ag}-\mathrm{Cl}$ distances were in the range $2.9-3.8 \AA{ }^{[18]}$ The SERS peaks of pyrene were resolved at $405,592,1135$, and $1234 \mathrm{~cm}^{-1}$, corresponding to the Raman shifts of pyrene standard. ${ }^{[20]} \mathrm{HA}$, the ubiquitous component in the environment, exhibited no significant SERS peaks and showed no influence on the identification of target hydrophobic pollutants [Fig. 5(I)]. However, a general decrease in peak intensity was observed because these three hydrophobic pollutants compete for the 'hot spots' on the Au NPs. In addition, aqueous matrices show no impact on the identification of these three pollutants [Fig. 5(II)]. The results verify the potential BDE-47 detection and identification in a wide environmental matrice. The identification of structurally similar hydrophobic pollutants enabled SERS a powerful tool in the environmental analysis and their toxicity assessment.
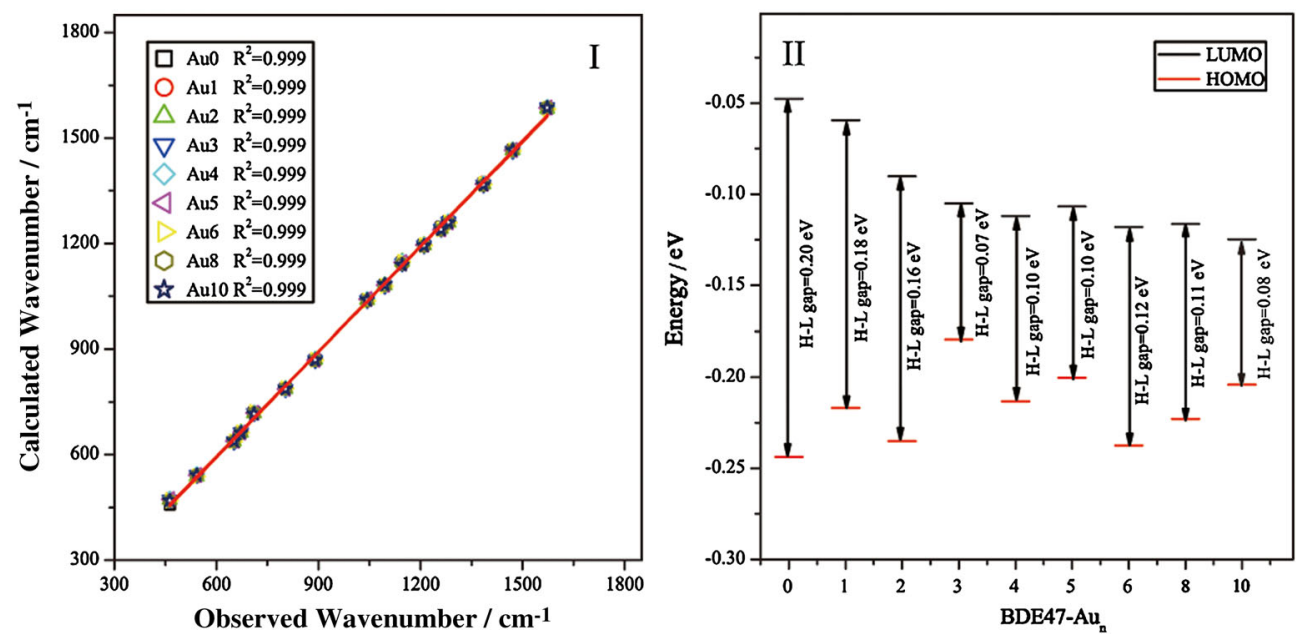

Figure 4. (I) Calibration curve for experimental and calculated peak position of BDE-47. (II) Variation of frontier orbital energies as the function of $\mathrm{BDE}-47 / \mathrm{Au}_{\mathrm{n}}$. 

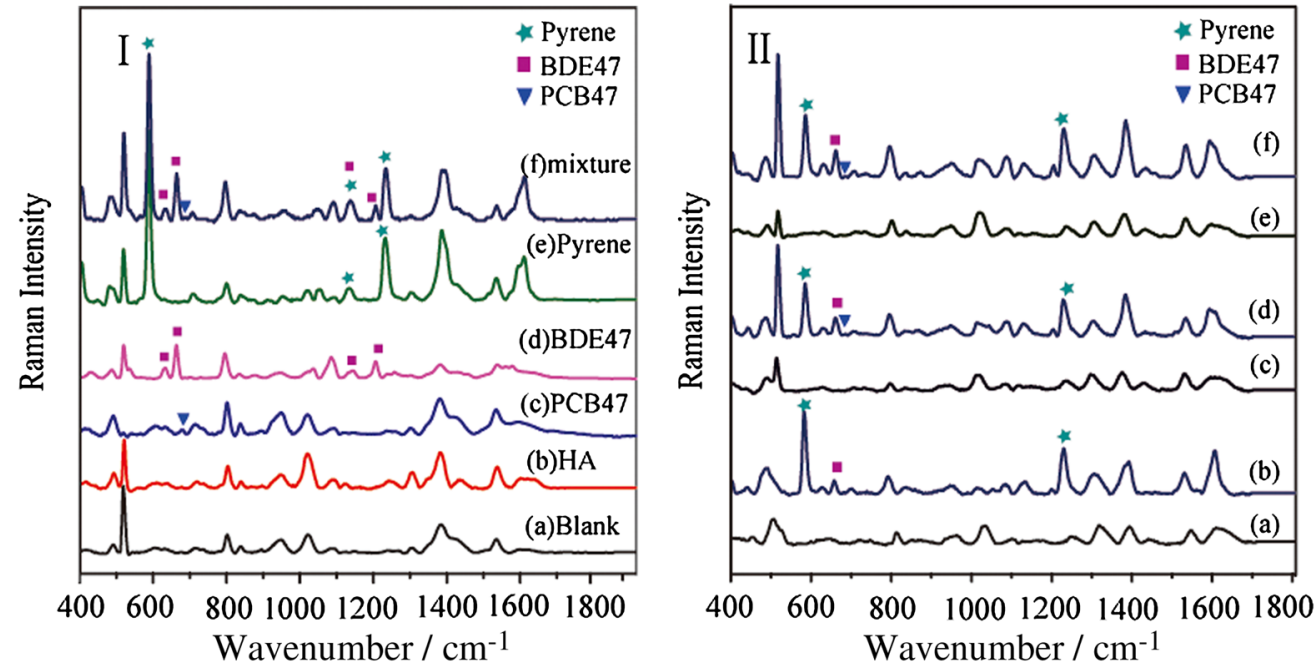

Figure 5. (I) SERS spectra in DI water for (a) Au colloid, (b) $1 \mathrm{mg} / \mathrm{L} \mathrm{HA}$, (c) $1 \times 10^{-6} \mathrm{M} \mathrm{PCB}-47$, (d) $1 \times 10^{-6} \mathrm{M}$ BDE- 47 , (e) $1 \times 10^{-6} \mathrm{M}$ pyrene, and (f) mixture of (b)-(e). (II) SERS spectra of the mixture and Au NPs blank in river water (b, a), groundwater (d, c), and tap water (f, e), respectively. Signal collecting time $=5 \mathrm{~s}$.

\section{Conclusions}

The present work demonstrates a facile method for the rapid detection of BDE-47. Qualitative and quantitative determination of BDE-47 with a detection limit at the 75-nM level was achieved, and the SERS response exhibited a linear dependence on BDE-47 concentrations between 75 and $1000 \mathrm{nM}$. The present approach does not require expensive instrumentation or specific functionalized NPs, which should have wide applications in the rapid identification of BDE-47 in complex samples. The sensitivity of this analytical method has opened a new way toward a generalized use of our technique not only in the laboratory but also in the field assays with a portable Raman spectrometer.

\section{Acknowledgements}

The research is supported by the Strategic Priority Research Program of the Chinese Academy of Sciences (XDB14020201), the Nature Science Foundation of China (21307147, 21321004), the National Basic Research Program of China (2014CB114402), the National Hi-tech Research Program of China (2011YQ0301241002), and RCEES (YSW2013A01).

\section{References}

[1] W. C. Du, R. Ji, Y. Y. Sun, J. G. Zhu, J. C. Wu, H. Y. Guo, Environ. Sci. Technol. 2013, 47, 9167.

[2] X. Gong, Y. Bao, C. Qiu, C. Y. Jiang, Chem. Commun. 2012, 48, 7003.

[3] P. White, J. Hjortkjaer, J. Raman Spectrosc. 2014, 45, 32.
[4] H. B. Tang, G. W. Meng, Q. Huang, Z. Zhang, Z. L. Huang, C. H. Zhu, Adv. Funct. Mater. 2012, 22, 218.

[5] X. F. Shi, Y. H. Kwon, J. Ma, R. E. Zheng, C. Y. Wang, H. D. Kronfeldt, J. Raman Spectrosc. 2013, 44, 41.

[6] J. J. Du, C. Y. Jing, J. Phys. Chem. C 2011, 115, 17829.

[7] X. H. Jiang, Y. C. Lai, W. Wang, W. Jiang, J. H. Zhan, Talanta 2013, 116, 14.

[8] K. C. Bantz, C. L. Haynes, Vib. Spectrosc. 2009, 50, 29.

[9] L. Guerrini, J. V. Garcia-Ramos, C. Domingo, S. Sanchez-Cortes, Anal. Chem. 2009, 81, 953.

[10] E. K. Beloglazkina, A. G. Majouga, R. B. Romashkina, N. V. Zyk, N. S. Zefirov, Russ. Chem. Rev. 2012, 81, 65.

[11] G. Maiorano, S. Sabella, B. Sorce, V. Brunetti, M. A. Malvindi, R. Cingolani, P. P. Pompa, ACS Nano 2010, 4, 7481.

[12] I. Blakey, Z. Merican, K. J. Thurecht, Langmuir 2013, 29, 8266.

[13] W. Haiss, N. T. K. Thanh, J. Aveyard, D. G. Fernig, Anal. Chem. 2007, $79,4215$.

[14] E. C. Le Ru, E. Blackie, M. Meyer, P. G. Etchegoin, J. Phys. Chem. C 2007, 111, 13794.

[15] C. H. Munro, W. E. Smith, M. Garner, J. Clarkson, P. C. White, Langmuir 1995, 11, 3712.

[16] G. S. Hu, Z. C. Feng, J. Li, G. Q. Jia, D. F. Han, Z. M. Liu, C. Li, J. Phys. Chem. C 2007, 111, 11267.

[17] L. L. Zhao, L. Jensen, G. C. Schatz, J. Am. Chem. Soc. 2006, 128, 2911.

[18] W. X. Pan, Y. C. Lai, R. X. Wang, D. J. Zhang, J. H. Zhan, J. Raman Spectrosc. 2014, 45, 54.

[19] Z. Mao, W. Song, X. Xue, W. Ji, Z. Li, L. Chen, H. Mao, H. Lv, X. Wang, J. R. Lombardi, B. Zhao, J. Phys. Chem. C 2012, 116, 14701.

[20] B. Sun, Z. A. Dreger, Y. M. Gupta, J. Phys. Chem. A 2008, 112, 10546.

\section{Supporting information}

Additional supporting information may be found in the online version of this article at the publisher's web site. 